\title{
Hydrocephaly and secondary intracranial hypertension to racemosa neurocysticercosis
}

\author{
Patrick Giordanni Gomes Sampaio (iD) Hiago Diniz Maracajá (iD) Túlio Carneiro Monteiro Temoteo (iD Sara \\ Raquel Nóbrega Figueiredo (D) Francielle Lopes de Araújo Batista
}

Unifacisa, Medicina, Campina Grande, Paraíba, Brazil

\section{$\bigotimes$}

Hiago Diniz Maracajá

hiago_maracaja@yahoo.com.br

Edited by:

Marcelo Moraes Valença

Keywords:

Hydrocephalus

Intracranial Hypertension

Neurocysticercosis

\begin{abstract}
Case report

G.F.M, 71 years old, male, farmer, presented a history of holocranial headache of a pulsatile type, more intense on waking, and associated with photophobia. The complaint lasted six months. He also reported episodes of tinnitus and unilateral hearing loss on the left. After conducting screening tests in the emergency department, communicating hydrocephalus and cystic lesions were found in the subarachnoid and intraparenchymal space. In the neurological examination, the positive data were bilateral papilledema and hearing loss in the left boné conduction. Given these findings, the racemous variant of neurocysticercosis and secondary hydrocephalus was suspected, causing intracranial hypertension. Thus, it was decided to start specific treatment with albendazole $15 \mathrm{mg} / \mathrm{kg} /$ day (two daily doses), associated with praziquantel $50 \mathrm{mg} / \mathrm{kg} /$ day (three daily doses), for 14 days. To reduce the inflammatory process of the lesions, it was proposed to keep the patient on dexamethasone $8 \mathrm{mg} /$ day for 90 days. In addition to this scheme, topiramate $100 \mathrm{mg} /$ day associated with acetazolamide $1 \mathrm{~g} /$ day was started, along with serial lumbar puncture to relieve intracranial pressure.

Conclusion

The combination of modern diagnostic tests, use of antiparasitic drugs, optimization of anti-inflammatory treatments and minimally invasive neurosurgical procedures have improved results in patients with NCC. Even so, this is the helminth infection that most affects the CNS and represents a major public health problem in most parts of the world, since it remains a neglected pathology, like so many other parasites, given that it is susceptible to containment through simple preventive actions as well as eradication.
\end{abstract}




\section{Introduction}

ysticercosis is an infection caused by the larvae of Taenia solium, which is an intestinal parasite of animals - like a pig - and has a secondary life cycle in humans. This is the parasitic infection that mostly affects the brain, at the time being called neurocysticercosis (NCC), and may infiltrate intra or extraparenchymal areas. ${ }^{1}$

NCC is highly endemic in most low-income countries, including areas such as Latin America, African Sahara, and parts of $\mathrm{Asia}^{2,3}$, being considered an emerging condition in developed countries in Europe, as well as in the USA and Canada, due to the migratory movements. It is estimated that between 1320 and 5050 new cases of NCC occur per year in the USA. ${ }^{4}$ In Latin America and the Caribbean, an estimated 75 million people are at risk of infection. In Brazil, the Southeast, South, and Midwest regions are considered endemic. A study presented in 2003 showed that the frequency of NCC cases ranged from $0.12 \%$ to $9 \%$ in autopsies and from $0.03 \%$ to $13.4 \%$ in clinical studies. The lethality rate ranged from $4.8-25.9 \%$, and asymptomatic forms were detected in $48-55 \%$ of cases with a diagnosis confirmed by autopsy. ${ }^{5}$

Racemic neurocysticercosis is a variant of the subarachnoid form of NCC. It is extremely rare and clinically more aggressive than the intraparenchymal form, characterized by the appearance of an image in the form of a grape cluster, the result of an aberrant proliferation of the cysticercar membrane with simultaneous degeneration of the scolex. Clinical manifestations include hydrocephalus, mass effect, chronic meningitis, seizure, stroke, reversible dementia, and cranial neuropathy. This variant accounts for $10 \%$ of cases. ${ }^{6}$ The diagnosis is established based on the clinical presentation and neuroimaging exams - computed tomography and magnetic resonance. ${ }^{7}$

\section{Case report}

G.F.M, 71 years old, male, farmer, presented a history of holocranial headache of a pulsatile type, more intense on waking, and associated with photophobia. The complaint lasted six months. He also reported episodes of tinnitus and unilateral hearing loss on the left. The case progressively evolved to the point that the patient complained of changes in visual acuity. After this new symptom, the patient developed a generalized tonic-clonic seizure and was then referred for hospital evaluation at a neurological referral service.
After conducting screening tests in the emergency department, communicating hydrocephalus and cystic lesions were found in the subarachnoid and intraparenchymal space. Laboratory tests showed no significant changes. In the neurological examination, the positive data were bilateral papilledema and hearing loss in the left bone conduction. Given these findings, the racemous variant of neurocysticercosis and secondary hydrocephalus was suspected, causing intracranial hypertension. As there were no signs of contraindication to lumbar puncture, this test was carried out, which presented an opening pressure of $38 \mathrm{cmH} 20$, confirming the presence of $\mathrm{ICH}$, cellularity $102 \mathrm{~mm} 3$, proteinorchia of $158 \mathrm{mg} / \mathrm{dL}$, hypoglycorrhea of $42 \mathrm{mg} / \mathrm{dL}$, and test from chemiluminescence to positive neurocysticercosis.

Thus, it was decided to start specific treatment with albendazole $15 \mathrm{mg} / \mathrm{kg} /$ day (two daily doses), associated with praziquantel $50 \mathrm{mg} / \mathrm{kg} /$ day (three daily doses), for 14 days. To reduce the inflammatory process of the lesions, it was proposed to keep the patient on dexamethasone 8 $\mathrm{mg} /$ day for 90 days. In addition to this scheme, topiramate $100 \mathrm{mg} /$ day associated with acetazolamide $1 \mathrm{~g} /$ day was started, along with serial lumbar puncture to relieve intracranial pressure.

The patient evolved with a satisfactory improvement of headache and low visual acuity and maintained the condition of hearing loss even after the treatment performed.

\section{Coments}

Some factors predispose to contagion, among them the underdevelopment and prevalence of the rural region with the absence of basic sanitary measures. 8,9 Neurocysticercosis is classified into extra and intraparenchymal forms. In the intraparenchymal form, seizures are almost pathognomonic for this condition. ${ }^{8,10}$ And in general, in up to $70 \%$ of patients, they represent the first manifestation of the disease and occur, mostly, due to the degeneration of the parasite or the release of antigens from the calcified cysticercus." ${ }^{11}$ Thus, it is justifiable that the main stages of cysticerci found by neuroimaging are the final stages, the granular stage, and the calcified stage. ${ }^{8,10}$ In many cases, there are recurrences of seizures, directly related to residual calcifications and, mainly, with hippocampal sclerosis. ${ }^{8}$

The extraparenchymal form includes the presence of cysts in the subarachnoid, intraventricular space, spinal cord, or 
intraocular space. In subarachnoid involvement, cysticerci may be present in the cortical grooves. However, if these cysts are located in the basal cisterns, they have a great chance of having the appearance of a "grape bunch", characterizing the racemic variant, whose main clinical manifestation - ICH - is caused by the "mass effect" of the cysts, obstructing CSF flow 9,11 and causing non-obstructive hydrocephalus, with nocturnal worsening and morning improvement, justified by the orthostatic position, which favors the drainage of cerebrospinal fluid (CSF).

According to the criteria of Del Brutto, it is possible to close a diagnosis through a biopsy of the lesion with histopathological study, the visualization of subretinal parasites by fundoscopy or the visualization of the cystic of the cysticercus in the neuroimaging. In the absence of these absolute criteria, it is possible to associate the image with epidemiological, clinical, and/or serological criteria. It is important to note that the ELISA is not the most suitable immunological test, due to the inconsistency in sensitivity and specificity, since intraparenchymal lesions are usually not enough to cause changes in the serology of the CSF sample. , $^{8,1,12}$

NCC presents a wide variety of possible differential diagnoses concerning its neuroimaging, the main ones being: benign and malignant tumors. Simulating the intraparenchymal form, it is necessary to remember, especially in the presence of single lesions, of brain tuberculomas, which, in addition to sharing endemic zones with the NCC, also present the "hole-in-dot" sign, previously considered to be pathognomonic of the NCC. Regarding the extraparenchymal form, cystic hemangioblastomas and colloid cysts should be excluded, which can also obstruct the CSF flow, mimicking $\mathrm{ICH}$ and headache present in some cases of NCC. ${ }^{13}$

About communicating hydrocephalus is initially indicated to perform ventricular shunt due to $\mathrm{ICH}$, and then to carry out conservative drug treatment. ${ }^{11}$ However, given the risk of bleeding during the procedure, socioeconomic conditions, and the not so exacerbated clinic, conservative treatment may be the first option. In the case in question, an assessment was requested from the neurosurgery team, which chose not to proceed with a peritoneal shunt, against what the literature recommends.

It is worth mentioning that the proposed treatment for the racemous form is not the typical treatment performed for NCC with single and mild lesions, in which only one antiparasitic can be prescribed, lasting an average of 10 to 14 days depending on the radiological resolution of the cyst. Generally, the treatment of racemosa is carried out by combining albendazole, $15 \mathrm{mg} / \mathrm{kg} /$ day, maximum dose of $1200 \mathrm{mg}$, divided into two doses, with praziquantel, $50 \mathrm{mg} / \mathrm{kg} /$ day divided into 3 doses, until radiological resolution of the condition, associated with the use of corticosteroids or methotrexate, as an alternative to prolonged treatment.7 This, in turn, requires monitoring of the patient's biochemical parameters, due to the potential hepatotoxic and leukopenic effects of this treatment.

Corticosteroids (Prednisone $1 \mathrm{mg} /$ day or dexamethasone $0.1 \mathrm{mg} /$ day) are used before antiparasitic therapy has been prescribed to reduce inflammation/edema of brain tissue caused mainly by cyst degeneration, resulting from antiparasitic treatment. ${ }^{13,14}$ In patients with subarachnoid cysts, mainly in the racemous form, as well as in those with multiple intraparenchymal, ventricular and medullary cysts, the administration of corticosteroids before and during antiparasitic therapy is mandatory, since, in addition to being necessarily longer, the inflammatory reaction caused by the destruction of the cyst can be exacerbated to the point of occluding vessels, causing cerebral infarctions, as well as massive cerebral edema."

When there is calcification of cysticerci, antiparasitic therapy is no longer necessary, as the cysts are no longer viable. Also, the prescription of corticosteroids is not indicated even in the presence of perilesional edema, to avoid rebound effect at the end of this treatment. ${ }^{14}$

\section{Conclusion}

From the report presented and the association between the radiological images and the presented clinical picture, the diagnosis of the racemic variant of neurocysticercosis was reached.

This is the helminth infection that most affects the CNS and represents a major public health problem in most parts of the world, since it remains a neglected pathology, like so many other parasites, given that it is susceptible to containment through simple preventive actions, as well as eradication. This, in turn, depends on individualized clinical treatment, with increased time for subarachnoid cysts (mainly in the racemous variant), use of corticosteroids before and concomitant with the use of antiparasitic drugs in most cases, and use of anticonvulsants when necessary. For this, a diagnosis based on neuroimaging is essential but supplemented by the individual's epidemiology, clinic, and even serology. 
Financing source: No

Conflict of interests: No

Authors' contribution: PGGS - Data Collection, Preparation and Writing of the Original; HDM and FLAB - Methodology, Preparation and Writing of the Original; TCMT and SRNF- Writing of the Original

Patrick Giordanni Gomes Sampaio https://orcid.org/0000-0003-0952-9798 Hiago Diniz Maracajá https://orcid.org/0000-0002-5032-3436 Túlio Carneiro Monteiro Temoteo https://orcid.org/0000-0001-6160-3321 Sara Raquel Nóbrega Figueiredo https://orcid.org/0000-0002-3832-4065 Francielle Lopes de Araújo Batista https://orcid.org/0000-0002-7256-4070

\section{References}

1. Frackowiak $M$, Sharma $M$, Singh $T$, Mathew $A$ and Michael BD. Antiepileptic drugs for seizure control in people with neurocysticercosis. Cochrane Database Syst Rev 2019;10(10):Cd009027 Doi: 10.1002/14651858.CD009027.pub3

2. Coyle CM. Neurocysticerosis: An Individualized Approach. Infect Dis Clin North Am 2019;33(1):153168 Doi: 10.1016/i.idc.2018.10.007

3. White Jr AC, Coyle CM, Rajshekhar V, Singh G, Hauser WA, Mohanty A, . . Nash TE. Diagnosis and treatment of neurocysticercosis: 2017 clinical practice guidelines by the Infectious Diseases Society of America (IDSA) and the American Society of Tropical Medicine and Hygiene (ASTMH). J Clinical Infectious Diseases 2018;66(8):e49-e75 Doi: 10.1093/cid/ cix 1084

4. Hong BY, Bean A, Vickers A, Cykowski M and Lee AG. Fourth Nerve Palsy Due to Neurocysticercosis. J
Neuroophthalmol 2020;40(3):414-416 Doi: 10.1097/wno.0000000000000785

5. Krupa K, Krupa K, Pisculli ML, Athas DM and Farrell CJ. Racemose neurocysticercosis. Surg Neurol Int 2016;7:12 Doi: 10.4103/2152-7806.175881

6. Costa PS, Santiago IG, Lima WR, Santos CS, Rocha $\mathrm{AV}$ and Fortes PM. Racemose neurocysticercosis: a cluster of bad grapes. Int J Infect Dis 2016;48:5-6 Doi: 10.1016/i.ijid.2016.04.012

7. McClugage SG, Lee RA, Camins BC, Mercado-Acosta $\mathrm{JJ}$, Rodriguez $M$ and Riley KO. Treatment of racemose neurocysticercosis. Surg Neurol Int 2017;8:168 Doi: 10.4103/sni.sni_157_17

8. White $A C$, Jr. and Garcia HH. Updates on the management of neurocysticercosis. Curr Opin Infect Dis 2018;31(5):377-382 Doi: 10.1097/ qco.0000000000000480

9. Gripper LB and Welburn SC. Neurocysticercosis infection and disease-A review. Acta Trop 2017;166:218-224 Doi: 10.1016/i. actatropica.2016.11.015

10. Delgado-García G, Méndez-Zurita VA, Bayliss L, Flores-Rivera J and Fleury A. Neurocysticercosis: mimics and chameleons. Pract Neurol 2019; 19(2):8895 Doi: 10.1136/practneurol-2017-001788

11. $P N$ and $G$ B. Handbook of Clinical Neurology: Neurologic Aspects of Systemic Disease Part III. Elsevier 2014;121(97):1445 -1459

12. Reddy DS and Volkmer R, 2nd. Neurocysticercosis as an infectious acquired epilepsy worldwide. Seizure 2017;52(1)176-181 Doi: 10.1016/i. seizure.2017.10.004

13. Garcia HH. Neurocysticercosis. Neurol Clin 2018;36(4):851-864Doi: 10.1016/j.ncl.2018.07.003

14. White AC. Cysticercosis: Treatment [Intemet]. UpToDate. 2019 [cited2020Dec9].Availablefrom: hittps://www.uptodate.com/ contents/cysticercosistreatment? search=neurocisticercose $\% 20$ racemosa\&source=search_result\&selectedTitle=3 20\&usage_ type=default\&display_rank=3\#H1312982321. 